The illustration shows the mirror image forms of $\mathrm{L}$ - and D-methionine with Vibrio cholerae and was based on an image provided by H. Lam, Brigham and Women's Hospital, Boston, USA.

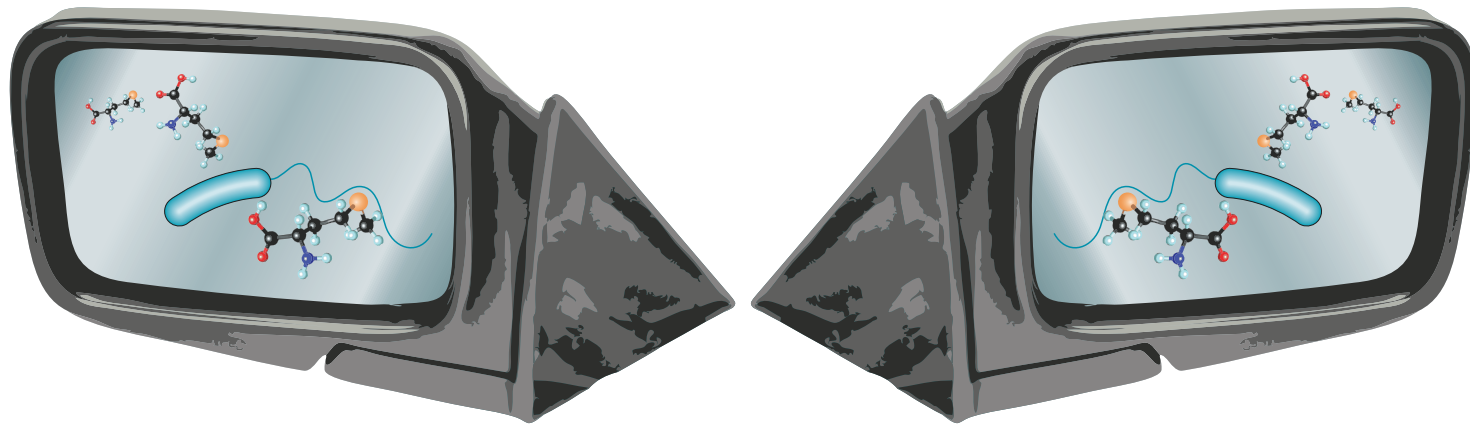

BACTERIAL PHYSIOLOGY

\title{
Mirror signal, manoeuvre
}

A recent Science paper from the Waldor laboratory provides evidence that bacteria use $\mathrm{D}$-amino acids to remodel the cell wall during the adaptation to stationary phase.

Almost all naturally occurring proteinogenic amino acids are chiral and, as such, can exist as L- and $D$-form mirror-image stereoisomers. In nature, $\mathrm{L}$-forms predominate, and few biological functions have been assigned to $\mathrm{D}$-amino acids. In bacteria, however, two D-amino acids, D-Ala and D-Glu, are incorporated into the cell wall peptidoglycan. Lam, Oh, Cava and colleagues were investigating cell wall synthesis in Vibrio cholerae when they noticed that $V$. cholerae cells with a mutation in one of the penicillin-binding proteins involved in peptidoglycan synthesis underwent an unusual morphological transition from rod-shaped to coccoid cells during stationary phase. This switch was controlled by a soluble factor or factors that are secreted into the culture supernatant. Fractionation of stationary-phase supernatants revealed the presence of D-amino acids, and a time course analysis showed that these amino acids accumulated in the supernatant to millimolar levels during stationary phase. D-Ala and D-Glu are known to be produced from their L-form enantiomers by an amino acid racemase.
Lam et al. identified a gene encoding a periplasmic racemase in $V$. cholerae, $v c 1312$, which they renamed $b s r V$ (broad-spectrum racemase Vibrio). $\mathrm{D}$-amino acid production in $\Delta b s r V$ cells was greatly reduced compared with production in wild-type cells.

As cells enter stationary phase they shut down unnecessary metabolic pathways. So why divert resources to the production of $\mathrm{D}$-amino acids? The authors looked at the composition of the peptidoglycan in wild-type and $\Delta b s r V V$. cholerae and found that, although there were no differences during exponential growth, $\Delta b s r V$ stationary-phase cells contained twice as much peptidoglycan as wild-type stationary-phase cells. There were also differences in peptidoglycan structure, and peptidoglycan from $\Delta b s r V$ cells was less resistant to osmotic stress than that from wild-type $V$. cholerae cells. The authors conclude that the production of $\mathrm{D}$-amino acids by the $\mathrm{BsrV}$ racemase regulates the amount and composition of peptidoglycan in V. cholerae stationary-phase cells. The mechanisms involved remain to be fully determined, but high-performance liquid chromatography analysis revealed that D-Met could be incorporated into $\mathrm{V}$. cholerae peptidoglycan. Further work suggested that $\mathrm{D}$-amino acids might also regulate the activity of the penicillin-binding proteins that are responsible for peptidoglycan synthesis and modification.

Finally, Lam et al. showed that this phenomenon is not restricted to $V$. cholerae. The production of $\mathrm{D}$-amino acids and the presence of putative racemases were observed in many other species, including Bacillus subtilis and Staphylococcus aureus. More detailed analysis of the effects of $\mathrm{D}$-amino acid production on B. subtilis stationary-phase cultures showed that $\mathrm{D}$-amino acids also downregulate peptidoglycan synthesis in this species.

These results suggest that bacteria use $\mathrm{D}$-amino acids to regulate the cell wall remodelling that is required on entry to stationary phase, and the authors propose that D-amino acids could be used to synchronously control this process in a bacterial population. Further work is required to elucidate the details of the mechanisms involved, and this work shows that there is still much to be uncovered about the roles of $\mathrm{D}$-amino acids in bacteria.

\section{Sheilagh Molloy}

ORIGINAL RESEARCH PAPER Lam, H. et al. D-Amino acids govern stationary phase cell wall remodelling in bacteria. Science 325, 1552-1555 (2009) 